\title{
Le VIH au Canada - rapport de surveillance, 2019
}

\author{
Nisrine Haddad ${ }^{1}$, Ashley Weeks ${ }^{1}$, Anita Robert ${ }^{1}$, Stephanie Totten ${ }^{1}$
}

\section{Résumé}

Contexte : Le virus de l'immunodéficience humaine $(\mathrm{VIH})$ est un problème de santé publique mondial. Au Canada, le VIH doit être déclaré au niveau national depuis 1985. L'Agence de la santé publique du Canada (l'Agence) surveille les tendances des nouveaux diagnostics de VIH.

Objectifs : L'objectif de ce rapport de surveillance est de donner un aperçu de l'épidémiologie des cas de VIH déclarés en 2019 au Canada. Le rapport met en évidence les tendances sur 10 ans (2010 à 2019). Des données sur les diagnostics de VIH établis lors des examens médicaux aux fins de l'immigration et les tendances en matière de transmission périnatale du VIH sont également présentées.

Méthodes : L'Agence surveille le VIH au moyen du système national de surveillance du VIH/ sida, un système passif fondé sur les cas, qui recueille des données non nominatives soumises volontairement par toutes les provinces et tous les territoires canadiens. Des analyses épidémiologiques descriptives ont été effectuées sur les données nationales. Les données des examens médicaux aux fins de l'immigration ont été obtenues auprès d'Immigration, Réfugiés et Citoyenneté Canada, et les données sur les grossesses exposées au VIH ont été obtenues par l'entremise du Programme de surveillance périnatale du VIH au Canada.

Résultats : En 2019, 2122 diagnostics de VIH ont été signalés au Canada (5,6 pour 100000 habitants). La Saskatchewan a enregistré le taux de diagnostic provincial le plus élevé, soit 16,9 pour 100000 habitants. Le groupe des 30 à 39 ans a présenté le taux le plus élevé de diagnostic du VIH, soit 12,7 pour 100000 habitants. Alors que les taux pour les hommes et les femmes ont fluctué au cours de la dernière décennie, depuis 2010, les taux chez les hommes ont globalement diminué, tandis que le taux chez les femmes a légèrement augmenté. Comme les années précédentes, le taux de diagnostic chez les hommes en 2019 était plus élevé que chez les femmes (7,9 contre 3,4 pour 100000 habitants, respectivement). La proportion la plus élevée de tous les cas d'adultes signalés ayant une exposition connue était celle des gais, bisexuels et autres hommes ayant des rapports sexuels avec des hommes (gbHARSAH, $39,7 \%)$, suivie des cas attribués à des contacts hétérosexuels $(28,3 \%)$ et parmi les utilisateurs de drogues par injection (UDI, 21,5\%). Le nombre de migrants qui se sont révélés séropositifs au VIH lors d'un examen médical aux fins de l'immigration effectué au Canada était de 626. Le seul cas documenté de transmission du VIH durant la période périnatale concernait une mère qui n'avait pas reçu de traitement antirétroviral prophylactique antepartum ou intrapartum.

Conclusion : Le nombre et le taux de cas de VIH déclarés au Canada sont restés relativement stables au cours de la dernière décennie, avec des variations mineures d'une année à l'autre. Comme les années précédentes, les hommes gbHARSAH et les utilisateurs de drogues par injection représentent une proportion élevée des diagnostics de $\mathrm{VIH}$, bien qu'un nombre important de cas ait été attribué à des contacts hétérosexuels. II est important de surveiller régulièrement les tendances en matière de $\mathrm{VIH}$ en lien avec les engagements pancanadiens visant à réduire les répercussions sur la santé des infections transmissibles sexuellement et par le sang d'ici 2030.
Citation proposée : Haddad N, Weeks A, Robert A, Totten S. Le VIH au Canada - rapport de surveillance, 2019. Relevé des maladies transmissibles au Canada 2021;47(1):87-98. https://doi.org/10.14745/ccdr.v47i01a11f

Mots-clés : VIH, surveillance, hommes homosexuels, bisexuels et autres hommes ayant des rapports sexuels avec des hommes, contact hétérosexuel, utilisateurs de drogues par injection, VIH périnatal, Canada
Cette oeuvre est mise à la disposition selon les termes de la licence internationale Creative Commons Attribution 4.0

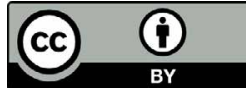

\section{Affiliation}

${ }^{1}$ Centre de la lutte contre les maladies transmissibles et les infections, Agence de la santé publique du Canada, Ottawa, ON

\section{Correspondance :}

phac.hass.aspc@canada.ca 


\section{Introduction}

Le virus de l'immunodéficience humaine $(\mathrm{VIH})$ a de graves répercussions économiques et sociales à l'échelle mondiale; on estime que 1,7 million de personnes dans le monde ont été nouvellement infectées par le VIH en 2019 (1). Au Canada, des estimations récentes indiquent qu'environ 62050 personnes vivaient avec le VIH à la fin de 2018. Parmi les personnes vivant avec le $\mathrm{VIH}, 87 \%$ ont reçu un diagnostic; $85 \%$ des personnes qui ont reçu un diagnostic recevaient un traitement, et $94 \%$ des personnes qui recevaient un traitement avaient une charge virale indétectable (2).

Des progrès plus récents dans les soins du $\mathrm{VIH}$, y compris la prophylaxie pré-exposition avec le traitement antirétroviral, et la disponibilité de l'autotest ont le potentiel d'avoir un impact considérable sur l'incidence du VIH au Canada. Malgré ces progrès, les personnes vivant avec le $\mathrm{VIH}$ sont confrontées à des défis importants, comme les obstacles pour obtenir des soins efficaces, les problèmes de santé tout au long de la vie liés au diagnostic de $\mathrm{VIH}$ ou à son traitement, ainsi que la stigmatisation et la discrimination (3).

Dans le cadre d'un mouvement mondial visant à éliminer les infections transmissibles sexuellement et par le sang (ITSS) en tant que problème de santé d'ici 2030, l'Agence de la santé publique du Canada (l'Agence) a publié un document intitulé Réduction des répercussions sur la santé des infections transmissibles sexuellement et par le sang au Canada d'ici 2030 : un cadre d'action pancanadien sur les ITSS (4). Ce cadre et le plan d'action (5) du gouvernement du Canada qui lui sont associés témoignent d'un engagement à réduire le fardeau des ITSS au Canada grâce aux piliers fondamentaux que sont la prévention, le dépistage, l'initiation des soins et des traitements, ainsi que les soins et le soutien continus. En outre, le cadre définit l'importance d'une approche commune pour traiter les populations touchées de manière disproportionnée par le VIH $(4,5)$. Le cadre souligne également l'importance d'un diagnostic et d'une déclaration précoces du $\mathrm{VIH}$ pour suivre les tendances des nouveaux diagnostics afin de contribuer aux programmes de prévention et de soins et de les évaluer (6-9). II est important de surveiller les tendances en matière de $\mathrm{VIH}$ pour comprendre le fardeau du VIH au Canada et pour suivre les progrès du Canada dans la réalisation des objectifs du cadre des ITSS. En 2018, le taux de diagnostic national était de 6,2 pour 100000 habitants avec un total de 2296 diagnostics de $\mathrm{VIH}$ (10). II y a eu six transmissions durant la période périnatale, dont quatre ont été attribuées à des mères qui n'ont pas reçu de traitement antirétroviral. Au total, 696 migrants ont reçu des diagnostics positifs pour le VIH lors d'examens médicaux aux fins de I'immigration effectués au Canada (10).

Les objectifs de ce rapport de surveillance sont de fournir des mises à jour sur l'épidémiologie des cas de VIH déclarés au Canada de 2010 à la fin de 2019, par lieu géographique, groupe d'âge, sexe et catégorie d'exposition. De plus, des renseignements à jour sur les résultats des examens médicaux de dépistage du $\mathrm{VIH}$ dans le cadre de l'immigration et sur le nombre de nourrissons exposés au VIH par voie périnatale et séropositifs au VIH sont présentés.

\section{Méthodes}

\section{Sources de données}

Les données présentées dans ce rapport de surveillance du VIH proviennent de trois sources différentes : le Système national de surveillance du $\mathrm{VIH} /$ sida de l'Agence, le dépistage médical du $\mathrm{VIH}$ aux fins d'immigration provenant d'Immigration, Réfugiés et Citoyenneté Canada et le Programme de surveillance périnatale du VIH au Canada. Des détails sur chaque source de données sont présentés ci-dessous.

\section{Système national de surveillance du VIH/sida}

Le Système national de surveillance du $\mathrm{VIH} /$ sida est un système de surveillance passif, basé sur les cas, qui recueille des données non nominales sur les personnes séropositives dont le cas respecte la définition nationale de cas (11). L'Agence reçoit des renseignements sur les éléments de données, notamment l'âge, le sexe, la race ou l'origine ethnique et les risques associés à la transmission du VIH (catégories d'exposition). Ces données sont volontairement fournies à l'Agence par les autorités provinciales et territoriales de santé publique.

Les données sur les catégories d'exposition et la race ou l'origine ethnique sont fournies avec des degrés variables d'exhaustivité dans tout le pays. Les données sur les catégories d'exposition ont été communiquées par toutes les provinces et tous les territoires à l'exception du Québec; par province et territoire, l'exhaustivité des données allait de $68,6 \%$ à $100 \%$ en 2019 (57,1\% dans l'ensemble). Les données sur la race ou l'origine ethnique ont été fournies par toutes les provinces et tous les territoires à l'exception du Québec et de la ColombieBritannique; pour les provinces et territoires qui ont communiqué des données sur la race ou l'origine ethnique, le taux de réponses complètes a varié entre $22 \%$ et $100 \%$ (41,5\% dans l'ensemble). Terre-Neuve-et-Labrador, le Yukon et le Nunavut ont fourni des renseignements sur la race ou l'origine pour tous les cas déclarés. En 2019, les Territoires du Nord-Ouest n'avaient pas de cas de diagnostic de VIH. En 2019, la Saskatchewan n'a fourni que deux sous-catégories de race ou origine ethnique, soit autochtone ou non autochtone. Le Nouveau-Brunswick n'a fourni qu'une seule sous-catégorie pour la catégorie de race ou origine ethnique, soit pour les personnes des Premières Nations, et n'a fourni aucun renseignement sur d'autres catégories de race ou origine ethnique.

Les données de chaque province et territoire sont obtenues par l'entremise des systèmes provinciaux de surveillance du VIH, qui peuvent inclure des rapports de santé publique et de laboratoire. 
Chaque province ou territoire fournit des données à l'Agence par l'entremise du formulaire de déclaration de cas (12) à l'échelle nationale ou par la transmission sécurisée d'un ensemble de données électroniques. Toutes les données brutes (formulaires papier et ensembles de données électroniques) sont conservées en conformité avec la Directive sur la collecte, l'utilisation et la diffusion de l'information sur la santé publique (Agence, 2013, document non publié). L'évaluation de la qualité des données, comme la détection des entrées en double, est réalisée par les provinces et les territoires qui les soumettent par la suite à l'Agence.

Les données présentées dans ce rapport de surveillance représentent les nouveaux cas de VIH diagnostiqués jusqu'au 31 décembre 2019 qui ont été présentés à l'Agence par les programmes de surveillance provinciaux et territoriaux jusqu'au 18 septembre 2020, et validés à partir du 8 octobre 2020 . Vous trouverez ailleurs d'autres détails sur les méthodes du système national de surveillance du VIH/sida (12).

L'Alberta et la Colombie-Britannique ont fourni à nouveau des données historiques révisées depuis 2016 et 2017, respectivement. Cette année, l'Ontario a fourni à nouveau des données historiques actualisées depuis 1985.

\section{Dépistage médical du VIH aux fins d'immigration}

Tous les ressortissants étrangers qui présentent une demande de résidence permanente au Canada et certains de ceux qui présentent une demande de résidence temporaire au Canada doivent subir un examen médical aux fins de l'immigration administré par des médecins tiers au nom d'Immigration, Réfugiés et Citoyenneté Canada, soit au Canada ou à l'étranger. Tous les candidats âgés de 15 ans et plus sont soumis à un test de dépistage du VIH lors de l'examen médical aux fins de I'immigration. Immigration, Réfugiés et Citoyenneté Canada fournit à l'Agence des données non nominales recueillies durant l'examen médical aux fins de l'immigration sur les migrants qui étaient séropositifs au VIH. Le terme migrant est utilisé au sens large et inclut notamment les immigrants (résidents permanents dans les catégories économique et familiale); les réfugiés (réfugiés réinstallés, personnes protégées et demandeurs d'asile); et les résidents temporaires (visiteurs, étudiants internationaux, travailleurs étrangers temporaires et titulaires de permis de séjour temporaire). Les données d'examens médicaux aux fins de l'immigration présentées ici proviennent du Système mondial de gestion des cas d'Immigration, Réfugiés et Citoyenneté Canada, mis à jour jusqu'en mars 2019, et qui contient l'information d'examens médicaux aux fins de l'immigration pour tous les candidats sélectionnés au Canada ou à l'étranger qui étaient séropositifs au VIH. Les données agrégées ont été fournies à l'Agence en juillet. Les données sur les personnes qui ont été testées au Canada ont été obtenues à partir d'examens médicaux aux fins de l'immigration réalisés en 2019. Les données concernant les personnes qui ont été testées à l'étranger ont été obtenues auprès de personnes ayant reçu un diagnostic de VIH dans le cadre de leur examen médical aux fins de l'immigration et qui sont arrivées au Canada en 2019.

Immigration, Réfugiés et Citoyenneté Canada partage avec les provinces et les territoires les données nominales des résultats des examens médicaux aux fins de l'immigration effectués à l'étranger pour tous les clients qui ont reçu un diagnostic de $\mathrm{VIH}$ et qui ont une adresse résidentielle canadienne valide dans leur dossier qui indique leur province ou leur territoire de résidence actuel. Cela favorise la continuité des soins pour les clients atteints du $\mathrm{VIH}$. Ces données sont intégrées à différents degrés, aux systèmes de surveillance provinciaux et territoriaux systématiques du VIH basés sur les cas; certains territoires et provinces considèrent ces cas de migrants séropositifs comme de nouveaux diagnostics alors que d'autres les excluent de la déclaration provinciale ou territoriale à l'Agence.

\section{Programme de surveillance périnatale du VIH au Canada}

Les données nationales sur la séropositivité des nourrissons exposés au VIH durant la période périnatale sont recueillies par le Programme de surveillance périnatale du VIH au Canada, une initiative du Groupe canadien de recherche sur le sida chez les enfants. Le Programme de surveillance périnatale du VIH au Canada est un système de surveillance sentinelle actif qui recueille des données sur deux groupes d'enfants : les nourrissons nés de femmes séropositives et les enfants séropositifs au VIH qui reçoivent des soins dans des sites participants (qu'ils soient nés au Canada ou à l'étranger). Des informations supplémentaires sur les méthodes du Programme de surveillance périnatale du VIH au Canada sont fournies ailleurs $(10,12)$. Les données de surveillance pour 2019, y compris les mises à jour des données pour les années précédentes, ont été soumises à l'Agence en mars 2020.

\section{Analyse}

Nous avons utilisé toutes les données sur les cas de VIH déclarés au système national de surveillance du $\mathrm{VIH} /$ sida pour effectuer des analyses descriptives des tendances générales, du lieu géographique, du sexe, de l'âge et des catégories d'exposition. Les analyses ont été limitées aux cas pour lesquels des données étaient disponibles (i.e. non manquantes). Les comptes et les proportions ont été calculés à partir des données d'Immigration, Réfugiés et Citoyenneté Canada. Le Programme de surveillance périnatale du VIH au Canada a fourni des tableaux de données agrégées, et certains résultats sont présentés dans ce rapport.

Les logiciels Excel 2016 de Microsoft (Redmond, Washington, États-Unis) et SAS Enterprise Guide v7.1 (Cary, Caroline du Nord, États-Unis) ont servi au nettoyage et à l'analyse des données. On a appliqué des procédures normalisées de recodage des données à tous les ensembles de données provinciales et territoriales soumises pour créer un ensemble de données nationales aux fins d'analyse. Dans ce rapport, le terme adulte 
est défini comme toute personne âgée de 15 ans ou plus. Les données de surveillance présentées dans ce rapport ont été validées par toutes les provinces et tous les territoires pour en assurer l'exactitude.

Aucune procédure statistique n'a été utilisée pour l'analyse comparative et l'on n'a appliqué aucune technique statistique pour tenir compte des données manquantes puisque les analyses étaient limitées aux tableaux croisés en raison de la nature descriptive de l'analyse. Les Statistiques démographiques annuelles, publiées par Statistique Canada en juillet 2018 (13), constituaient la source de données sur la population utilisée pour calculer les taux.

Les tableaux supplémentaires sont inscrits dans l'annexe et sont disponibles sur demande.

\section{Résultats}

\section{Tendances générales}

Au total, 88357 diagnostics de VIH ont été signalés à l'Agence depuis le début de la déclaration du VIH au Canada en 1985. En 2019, 2122 diagnostics de VIH ont été signalés. Le taux national de diagnostic était de 5,6 pour 100000 habitants. Ce taux a légèrement diminué depuis 2010, année à laquelle il était de 6,3 pour 100000 habitants (figure 1).

Figure 1 : Nombre de cas signalés de VIH, et les taux de diagnostic globaux, selon le sexe et l'année, Canada, 2010 à $2019^{a, b}$

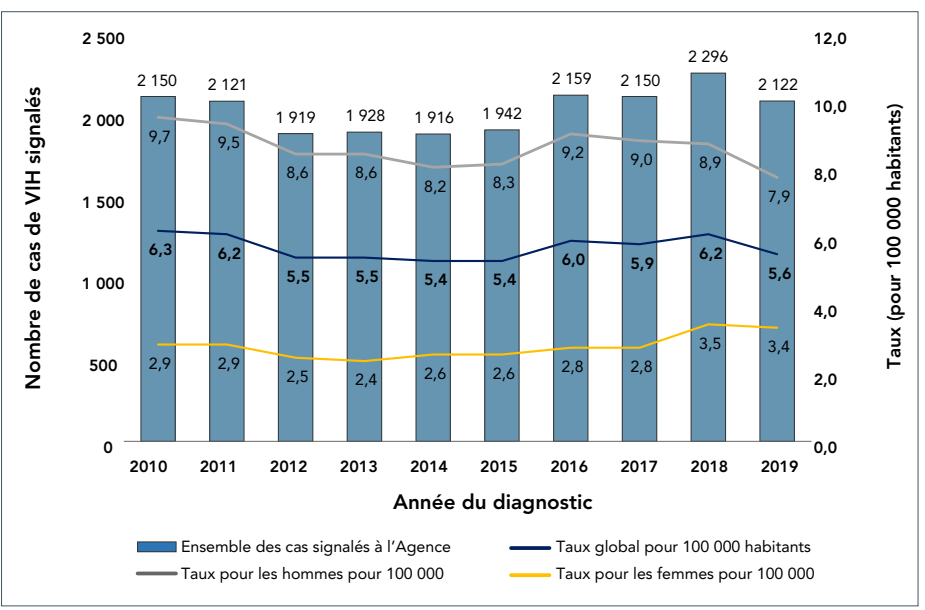

Abréviations : I'Agence, Agence de la santé publique du Canada; $\mathrm{VIH}$, virus de l'immunodéficience humaine

a Source de données sur la population : Statistiques démographiques annuelles, Division de la démographie, Statistique Canada, 1er juillet 2019

b Le taux global exclut les cas où le sexe est transgenre, transsexuel, non déclaré ou inconnu

En 2019, le taux de diagnostic était de 7,9 pour 100000 habitants chez les hommes et de 3,4 pour 100000 habitants chez les femmes. Alors que les taux pour les hommes et les femmes ont fluctué au cours de la dernière décennie, les taux dans la population masculine ont légèrement diminué depuis 2016 (de
9,2 à 7,9 pour 100000 habitants) et ont légèrement augmenté chez les femmes depuis 2015 (de 2,6 à 3,4 pour 100000 habitants) (figure 1).

\section{Distribution géographique}

En 2019, la Saskatchewan a enregistré le taux de diagnostic le plus élevé pour une province ou un territoire, soit 16,9 pour 100000 habitants. Le Manitoba a enregistré le deuxième taux de diagnostic le plus élevé pour une province ou un territoire, avec 8,8 pour 100000 habitants, suivi du Québec, de l'Alberta, de l'Ontario et de la Colombie-Britannique, avec respectivement $7,4,5,8,4,7$ et 3,5 pour 100000 habitants (figure 2 ).

Figure 2 : Taux de diagnostic du VIH (pour 100000 habitants) par province et territoire, Canada, 2019a,b

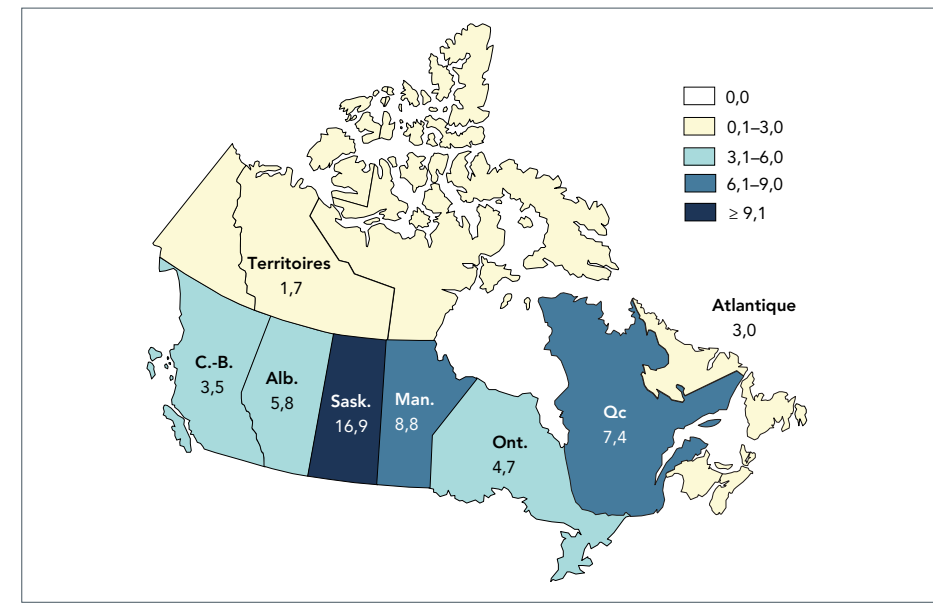

Abréviations : Alb., Alberta; C.-B., Colombie-Britannique; Man., Manitoba; Ont., Ontario Qc, Québec; Sask., Saskatchewan; VIH, virus de l'immunodéficience humaine a Les taux pour les territoires (Yukon, Nunavut et Territoires du Nord-Ouest) et la région de I'Atlantique (Île-du-Prince-Édouard, Nouveau-Brunswick, Nouvelle-Écosse et Terre-Neuve-etLabrador) sont présentés en moyennes

b Taux national de 5,6 cas pour 100000 habitants

\section{Répartition par groupe d'âge et par sexe}

En 2019, des données sur les groupes d'âge étaient disponibles pour près de $100 \%(n=2120)$ de tous les diagnostics de $\mathrm{VIH}$ déclarés. Le taux de diagnostic par groupe d'âge est resté stable depuis 2010 pour les personnes de moins de 19 ans et celles de 50 ans et plus. Le taux de diagnostic a légèrement fluctué au cours des dix dernières années pour les groupes d'âge de 20 à 29 ans, 30 à 39 ans et 40 à 49 ans. Le groupe de 30 à 39 ans a présenté le taux de diagnostic le plus élevé tout au long de la période de dix ans; en 2019, le taux était de 12,7 pour 100000 habitants, soit une diminution globale par rapport au taux de 14,3 pour 100000 habitants en 2010. La groupe d'âge de 20 à 29 ans se classe en deuxième position avec un taux de 10,1 pour 100000 habitants en 2019, suivi par le groupe d'âge de 40 à 49 ans avec un taux de 9,1 pour 100000 habitants. En 2019, le taux de diagnostic des personnes âgées de 50 ans et plus était de 3,2 pour 100000 habitants et celui des personnes âgées de 15 à 19 ans était de 1,6 pour 100000 habitants; les enfants de moins de 15 ans présentaient le taux de diagnostic le plus faible, soit 0,2 pour 100000 habitants (figure 3 ). 
Figure 3 : Taux de diagnostic du $\mathrm{VIH}$, tous les âges, par groupe d'âge et par année, Canada, 2010 à $2019^{a, b, c}$

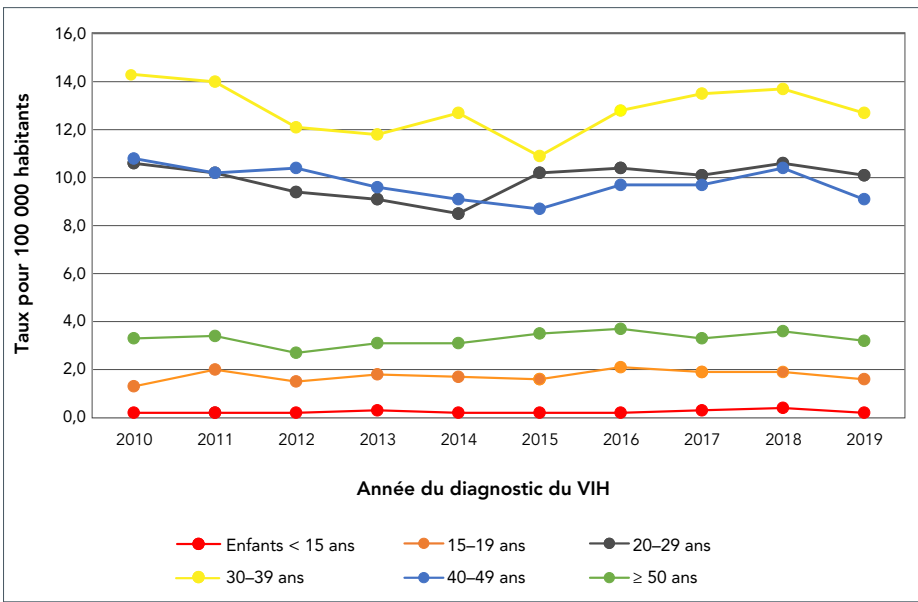

Abréviation : $\mathrm{VIH}$, virus de l'immunodéficience humaine

Exclut les cas pour lesquels le sexe est transsexuel, transgenre, non déclaré ou inconnu

b Exclut les cas pour lesquels l'âge n'est pas déclaré ou connu

Source de données sur la population: Statistiques démographiques annuelles, Division de la

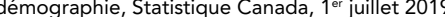

En 2019, des données sur le sexe étaient disponibles pour près de $100 \%(n=2118)$ de tous les diagnostics de VIH déclarés. Les hommes représentaient $69,8 \%$ des diagnostics pour lesquels le sexe était connu, tandis que les femmes représentaient $30,2 \%$.

Comme les années précédentes, les hommes âgés de 30 à 39 ans affichaient les taux de diagnostic les plus élevés en 2019 , avec 16,8 pour 100000 habitants; ce groupe d'âge présentait également les taux les plus élevés chez les femmes, avec 8,4 pour 100000 habitants. Chez les deux sexes, la plupart des diagnostics de VIH ont été posés chez les personnes âgées de 20 à 49 ans. Dans tous les groupes d'âge, à l'exception des moins de 19 ans, les taux chez les hommes étaient au moins deux fois plus élevés que chez leurs homologues féminins (figure 4).

Figure 4 : Taux de diagnostic du VIH, tous les âges, par sexe et groupe d'âge, Canada, 2019a,b,c

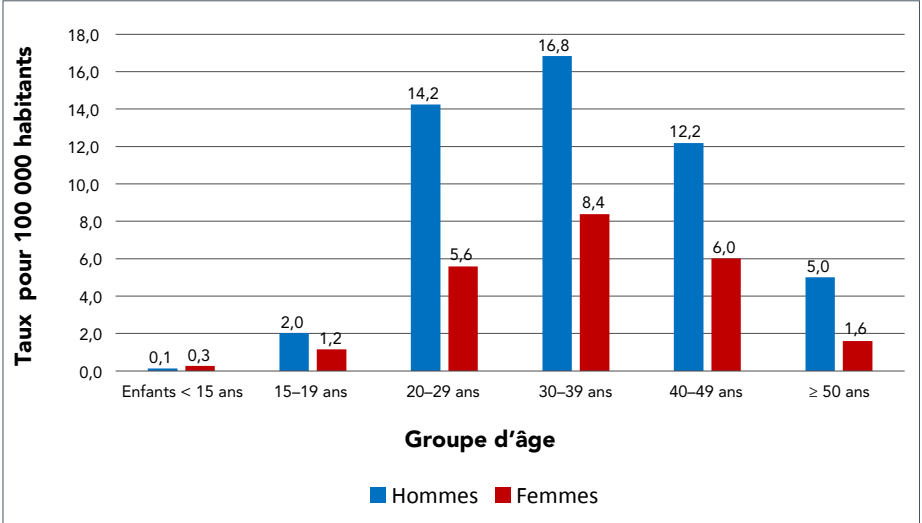

Abréviation : $\mathrm{VIH}$, virus de l'immunodéficience humaine

Exclut les cas pour lesquels le sexe est transsexuel, transgenre, non déclaré ou inconnu

b Exclut les cas pour lesquels l'âge n'est pas déclaré ou connu

'Source de données sur la population: Statistiques démographiques annuelles, Division de la démographie, Statistique Canada, 1 ${ }^{\text {er }}$ juillet 2019

\section{Race ou origine ethnique}

Les renseignements sur la race ou l'origine ethnique étaient connus pour 880 cas $(41,5 \%)$ en 2019 . Parmi les cas pour lesquels des renseignements sur la race ou l'origine ethnique étaient connus, 30,7 \% ont été signalés comme étant blancs, $25,5 \%$ comme étant noirs et $24,7 \%$ comme étant autochtones (Premières Nations, Inuits, Métis ou autochtones non précisés). La répartition des catégories de race ou l'origine ethnique varie selon le sexe; chez les hommes, la proportion la plus élevée a été signalée comme étant chez les blancs (38,5\%), tandis que les femmes ont été principalement signalées comme étant noires, à $42,1 \%$, et autochtones, à $40 \%$ (tableau 1 ).

\section{Tableau 1 : Distribution par nombre et répartition des cas de $\mathrm{VIH}$ (tous les âges) par sexe et race ou origine ethnique, Canada, 2019a,b}

\begin{tabular}{|c|c|c|c|c|c|c|}
\hline \multirow{3}{*}{$\begin{array}{l}\text { Race ou origine } \\
\text { ethnique }\end{array}$} & \multicolumn{4}{|c|}{ Cas de VIH } & \multirow{2}{*}{\multicolumn{2}{|c|}{ Total $^{c}$}} \\
\hline & \multicolumn{2}{|c|}{ Hommes } & \multicolumn{2}{|c|}{ Femmes } & & \\
\hline & $\mathbf{n}$ & $\%$ & $\mathbf{n}$ & $\%$ & $\mathbf{n}$ & $\%$ \\
\hline Autochtones & 104 & 17,4 & 112 & 40,0 & 217 & 24,7 \\
\hline Premières Nations & 45 & 7,5 & 46 & 16,4 & 92 & 10,5 \\
\hline Métis & 4 & 0,7 & 0 & 0,0 & 4 & 0,5 \\
\hline Inuit & 2 & 0,3 & 0 & 0,0 & 2 & 0,2 \\
\hline $\begin{array}{l}\text { Autochtones, non } \\
\text { précisés }\end{array}$ & 53 & 8,9 & 66 & 23,6 & 119 & 13,5 \\
\hline $\begin{array}{l}\text { Asie du Sud/Asie } \\
\text { occidentale/Arabe }^{d}\end{array}$ & 38 & 6,4 & 4 & 1,4 & 42 & 4,8 \\
\hline Asiatique $^{\mathrm{e}}$ & 42 & 7,0 & 3 & 1,1 & 45 & 5,1 \\
\hline Noir ${ }^{f}$ & 106 & 17,7 & 118 & 42,1 & 224 & 25,5 \\
\hline Latino-américaing & 62 & 10,4 & 2 & 0,7 & 64 & 7,3 \\
\hline Blanc & 230 & 38,5 & 39 & 13,9 & 270 & 30,7 \\
\hline Autre $^{h}$ & 16 & 2,7 & 2 & 0,7 & 18 & 2,0 \\
\hline Sous-total ${ }^{\mathrm{b}}$ & 598 & 40,5 & 280 & 43,8 & 880 & 41,5 \\
\hline Non déclaré & 880 & 59,5 & 359 & 56,2 & 1242 & 58,5 \\
\hline Total & 1478 & s.o. & 639 & s.o. & 2122 & s.o. \\
\hline
\end{tabular}

Abréviations : s.o., sans objet: $\mathrm{VIH}$, virus de l'immunodéficience humaine

Le Québec et la Colombie-Britannique ne communiquent pas d'informations sur la race ou l'origine ethnique; pour les autres provinces ou territoires, le taux de réponse varie, interprétez es données avec prudence

Tous les pourcentages sont calculés en utilisant la valeur du sous-total comme dénominateur ( $y$ compris uniquement les cas pour lesquels des données étaient disponibles)

'Le total comprend les cas où le sexe est transsexuel, transgenre et les cas où le sexe n'a pas été déclaré

dPar exemple, Arménien, Bangladais, Égyptien, Iranien, Libanais, Marocain, Pakistanais et

Sri-lankais

e Par exemple, Cambodgien, Chinois, Philippin, Indonésien, Japonais, Coréen, Laotien et Vietnamien

${ }^{f}$ Par exemple, Haïtien, Jamaïcain et Somalien

9 Par exemple, Centraméricain, Mexicain et Sud-Américain

Comprend les métis et toute autre catégorie

\section{Répartition des catégories d'exposition}

En 2019, 57,1 \% des diagnostics de VIH chez les adultes avaient une catégorie d'exposition connue $(n=1203)$. Comme pour les années précédentes, la proportion la plus élevée de tous les diagnostics de $\mathrm{VIH}$ chez les adultes en 2019 a été enregistrée chez les hommes gais, bisexuels et autres hommes ayant des rapports sexuels avec des hommes (gbHARSAH), soit 39,7\% ( $n=478$, bien que la proportion ait diminué au fil du temps, 
en particulier depuis 2015, où elle était de 45,0 \%. Les contacts hétérosexuels ont représenté $28,3 \%(n=340)$ des cas. Les sous-groupes de la catégorie des contacts hétérosexuels ont suivi une tendance constante, la proportion de contacts hétérosexuels sans risque identifié étant de 10,8 \% ( $n=130)$, suivie par les contacts hétérosexuels avec une personne d'un pays où le $\mathrm{VIH}$ est endémique à 9,2\% ( $n=111)$, et $8,2 \%(n=99)$ attribués aux contacts hétérosexuels avec une personne à risque. Les utilisateurs de drogues par injection représentaient 21,5\% $(n=259)$ des cas (tableau 2).

\section{Tableau 2 : Nombre et proportion de cas de VIH par sexe et catégorie d'exposition chez les adultes âgés de 15 ans ou plus, Canada (sauf le Québec), 2019a,b,c,d,e}

\begin{tabular}{|c|c|c|c|c|c|c|}
\hline \multirow{3}{*}{$\begin{array}{c}\text { Catégorie } \\
\text { d'exposition }\end{array}$} & \multicolumn{4}{|c|}{ Cas de VIH } & \multirow{2}{*}{\multicolumn{2}{|c|}{ Totale }} \\
\hline & \multicolumn{2}{|c|}{ Hommes } & \multicolumn{2}{|c|}{ Femmes } & & \\
\hline & $\mathbf{n}$ & $\%$ & $\mathbf{n}$ & $\%$ & $\mathbf{n}$ & $\%$ \\
\hline gbHARSAH & 477 & 56,2 & s.o. & s.o. & 478 & 39,7 \\
\hline gbHARSAH/UDI & 41 & 4,8 & s.o. & s.o. & 41 & 3,4 \\
\hline UDI & 124 & 14,6 & 135 & 38,4 & 259 & 21,5 \\
\hline Contact hétérosexuel & 170 & 20,0 & 169 & 48,0 & 340 & 28,3 \\
\hline Hét-Endémique & 36 & 4,2 & 75 & 21,3 & 111 & 9,2 \\
\hline Hét-Risque & 51 & 6,0 & 47 & 13,4 & 99 & 8,2 \\
\hline Hét-ARR & 83 & 9,8 & 47 & 13,4 & 130 & 10,8 \\
\hline Autre $^{a}$ & 37 & 4,4 & 48 & 13,6 & 85 & 7,1 \\
\hline Sous-total ${ }^{\mathrm{b}}$ & 849 & 100,0 & 352 & 100,0 & 1203 & 100,0 \\
\hline Aucun risque relevéc & 51 & 3,5 & 26 & 4,1 & 77 & 3,7 \\
\hline $\begin{array}{l}\text { Catégorie } \\
d^{\prime} \text { exposition inconnue } \\
\text { ou non signalée } \\
(« \text { manquante })^{d}\end{array}$ & 574 & 38,9 & 252 & 40,0 & 828 & 39,3 \\
\hline Total & 1474 & s.o. & 630 & s.o. & 2108 & s.o. \\
\hline $\begin{array}{l}\text { Abréviations : gbHARSAH, gais, } \\
\text { des hommes; Hét-ARR, contacts } \\
\text { hétérosexuels avec une personn } \\
\text { hétérosexuels avec une personn } \\
\text { injection; VIH, virus de l'immuno } \\
\text { 'Comprend les cas de l'Alberta } \\
\text { catégories de sang/produits san } \\
\text { d'exposition } \\
\text { ' Les proportions sont basées su } \\
\text { c Aucun risque relevé : Utilisé lor } \\
\text { autres modes énumérés sont in } \\
\text { (e.g. décès ou manque de suivi) } \\
\text { d Inclut tous les cas pour lesquel } \\
\text { le Québec n'a pas fourni d'infor } \\
\text { de VIH déclarés par le Québec s } \\
\text { "Le total inclut les cas de persor }\end{array}$ & $\begin{array}{l}\text { à risque } \\
\text { léficience } \\
\text { elevés pa } \\
\text { juins, exp } \\
\text { le total } \\
\text { que les a } \\
\text { onnus, ou }\end{array}$ & $\begin{array}{l}\text { o., sans } \\
\text { umaine } \\
\text { nmigrati } \\
\text { tion pér } \\
\text { iel de la } \\
\text { cédents } \\
\text { squ'auc }\end{array}$ & $\begin{array}{l}\text { endém } \\
\text { t; UDI, } \\
\text { Réfugi } \\
\text { ale, pro } \\
\text { égorie } \\
\text { xpositi } \\
\text { ntécéd } \\
\text { n n'éta } \\
\text { d'expo }\end{array}$ & $\begin{array}{l}\text { t des rapp } \\
\text { fié; Hét-En } \\
\text { ique; Hét- } \\
\text { utilisateurs } \\
\text { ss et Citoye } \\
\text { fessionnelle } \\
\text { d'expositio } \\
\text { on au VIH p } \\
\text { ent d'expos } \\
\text { it pas conn } \\
\text { sition, les n }\end{array}$ & $\begin{array}{l}\text { et autres } \\
\text { connue } \\
\text { r'un ou I } \\
\text { tion n'a é }\end{array}$ & $\begin{array}{l}\text { avec } \\
\text { ntacts } \\
\text { cts } \\
\text { par } \\
\text { da, } \\
\text { atégories } \\
\text { utre des } \\
\text { signalé } \\
\text { e. Comme } \\
\text { gnostics } \\
\text { ur lesquels }\end{array}$ \\
\hline
\end{tabular}

L'analyse de la variable de la catégorie d'exposition a été effectuée séparément pour les hommes et les femmes.

Parmi les hommes adultes en 2019, les hommes gbHARSAH représentaient la plus grande proportion $(56,2 \%, \mathrm{n}=477)$ des cas déclarés. Chez les femmes adultes, I'exposition par contact hétérosexuel représentait la proportion la plus élevée, soit $48,0 \%(n=169)$, avec $21,3 \%$ de contacts hétérosexuels avec une personne d'un pays où le VIH est endémique $(n=75)$ et $13,4 \%$ de contacts hétérosexuels avec une personne à risque et de contacts hétérosexuels sans risque identifié ( $n=47$ chacun).
En outre, les utilisateurs de drogues par injection représentaient un peu plus d'un tiers des cas de VIH chez les femmes adultes $(38,4 \%, n=135)$ contre $14,6 \%(n=124)$ des cas chez les hommes adultes (tableau 2).

La figure 5 montre la répartition des catégories d'exposition chez les hommes et les femmes au cours des dix dernières années. Chez les hommes, la répartition des diagnostics de $\mathrm{VIH}$ dans les différentes catégories d'exposition a légèrement fluctué depuis 2010, mais est restée relativement stable dans l'ensemble. II convient de noter que les catégories d'exposition pour les hommes gbHARSAH et hétérosexuelle ont légèrement diminué au cours des dix dernières années (diminution en pourcentage de $26,3 \%$ et $27,0 \%$, respectivement), tandis que la catégorie d'exposition pour les utilisateurs de drogues par injection est restée relativement stable. L'exposition attribuée à la catégorie gbHARSAH/utilisateurs de drogues par injection a augmenté au cours des 10 dernières années (augmentation en pourcentage de 10,9\%). Chez les femmes, on a constaté une diminution considérable de l'exposition attribuée aux contacts

Figure 5 : Répartition en pourcentage des cas de VIH chez (a) les hommes et (b) les femmes (15 ans ou plus) par catégorie d'exposition et année de diagnostic, Canada, 2010 à 2019 a,b

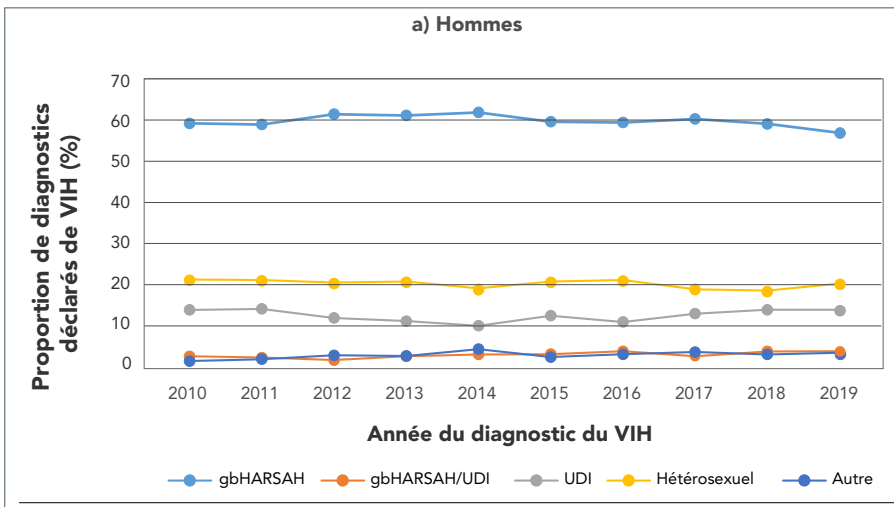

b) Femmes

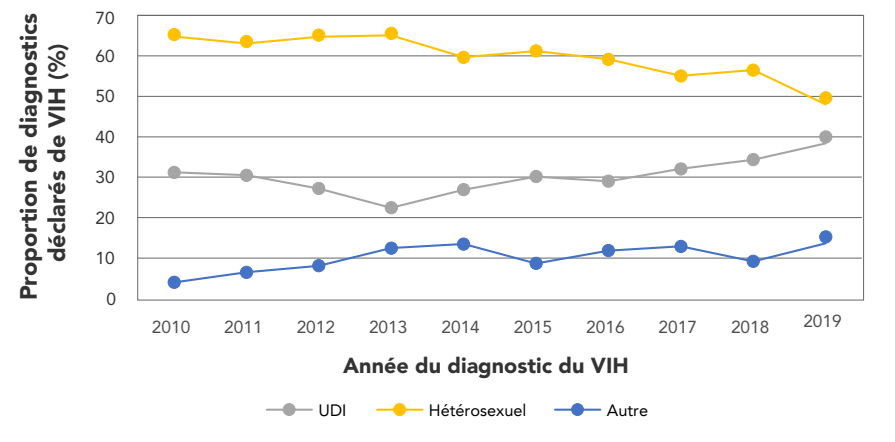

Abréviations : gbHARSAH, hommes gais, bisexuels et autres hommes ayant des rapports sexuels avec des hommes; gbHARSAH/UDI, hommes gays, bisexuels et autres hommes ayant des rapports sexuels avec des hommes et utilisateurs de drogues par injection; UDI, utilisateurs de drogues par injection; $\mathrm{VIH}$, virus de l'immunodéficience humaine

a Exclut les cas sans risque relevé, la catégorie d'exposition inconnue et les cas signalés par le Québec

${ }^{b}$ Autres comprend les cas de l'Alberta relevés par Immigration, Réfugiés et Citoyenneté Canada catégories de sang/produits sanguins, exposition périnatale, professionnelle et autres catégories d'exposition 
hétérosexuels (diminution de $20,3 \%$ en pourcentage), alors que la proportion des utilisateurs de drogues par injection a augmenté (augmentation de $32,4 \%$ en pourcentage).

\section{Répartition des catégories d'exposition par tranche d'âge}

En 2019, parmi les diagnostics de VIH dont la catégorie d'exposition est connue, la plus forte proportion de gbHARSAH et de gbHARSAH/utilisateurs de drogues par injection, se situait dans le groupe des 20 à 29 ans, avec respectivement 35,1\% $(n=168)$ et $41,5 \%(n=17)$. Parmi les utilisateurs de drogues par injection, la proportion la plus élevée $(35,1 \%, \mathrm{n}=91)$ se situait dans la tranche d'âge des 30 à 39 ans. Les cas signalés dans la catégorie d'exposition par contact hétérosexuel étaient également répartis entre les groupes d'âge différents pour les personnes âgées de plus de 20 ans (étendue : 21,5 à 26,8 \%), la proportion la plus élevée se situant dans le groupe d'âge des 30 à 39 ans (figure 6).

Figure 6 : Proportion des cas déclarés de VIH (âgés de 15 ans ou plus) par catégorie d'exposition et groupe d'âge, Canada, 2019a,b,c

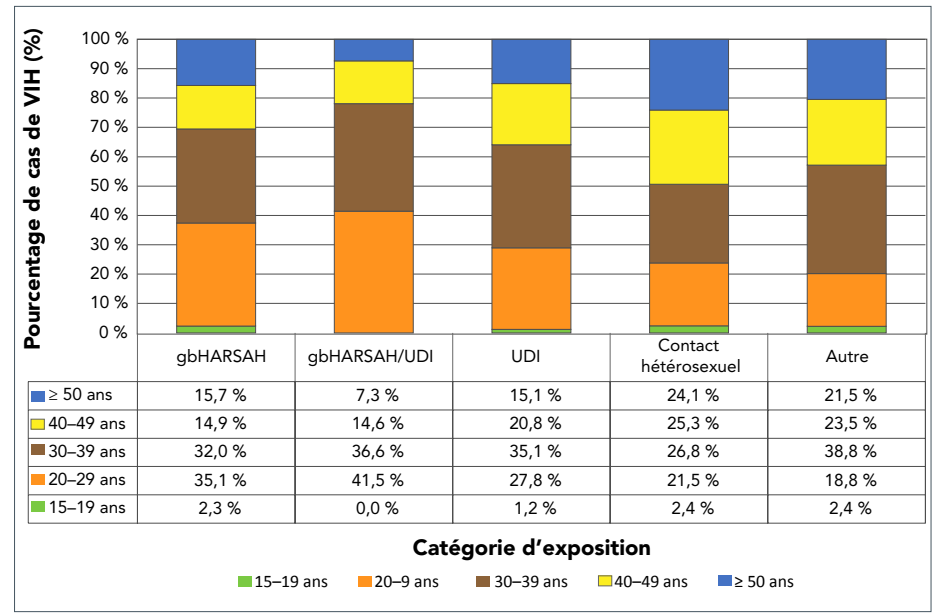

Abréviations : gbHARSAH, hommes gais, bisexuels et autres hommes ayant des rapports sexuels avec des hommes; gbHARSAH/UDI, hommes gays, bisexuels et autres hommes ayant des rapports sexuels avec des hommes et utilisateurs de drogues par injection; UDI, utilisateurs de drogues par injection; VIH, virus de l'immunodéficience humaine

a Comprend les cas où le sexe est transsexuel, transgenre et non déclaré

Exclut tous les cas pour lesquels la catégorie d'exposition n'était pas signalée

'Autres catégories d'exposition comprend les cas de l'Alberta relevés par Immigration

Réfugiés et Citoyenneté Canada, catégories de sang/produits sanguins, exposition périnatale professionnelle et autres categories d'exposition

\section{Dépistage médical du VIH aux fins d'immigration}

Entre 2010 et 2019, un total de 4090 personnes ont reçu un diagnostic positif pour le VIH lors d'un examen médical aux fins de l'immigration mené au Canada, soit une moyenne de 409 par an (étendue : 210-696) (figure 7). Au total, 1188 migrants ont reçu un diagnostic positif pour le VIH lors d'un examen médical aux fins de l'immigration en 2019. Parmi ces cas, $52,7 \%(n=626)$ ont été testés au Canada et $47,3 \%(n=562)$ ont été testés à l'étranger avant leur arrivée au Canada.
Figure 7 : Nombre de migrants qui se sont révélés séropositifs durant un examen médical aux fins de I'immigration effectué au Canada, par année de test, 2010 à 2019

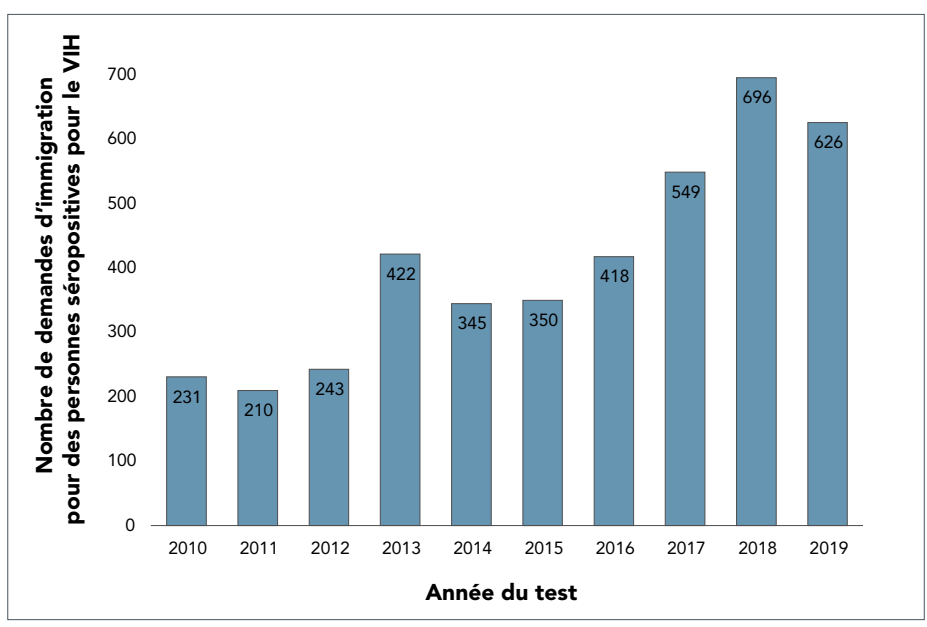

Abréviation : VIH, virus de l'immunodéficience humaine

En 2019, parmi les participants testés au Canada, une proportion légèrement plus élevée d'hommes $(54,6 \%$ ) que de femmes ont reçu un diagnostic positif lors d'un examen médical aux fins de l'immigration. Le groupe d'âge de 30 à 39 ans a enregistré la plus forte proportion de tests positifs $(36,1 \%)$, suivis de du groupe de 40 à 49 ans (26,8\%). Les candidats séropositifs au VIH de moins de 29 ans représentaient $22,2 \%$ du total, alors que ceux de plus de 50 ans ne représentaient que 14,9\% des candidats séropositifs au VIH testés au Canada. La majorité des candidats séropositifs au VIH au Canada se trouvaient en Ontario $(57,0 \%)$ et au Québec (24,6\%). Parmi les candidats séropositifs au VIH testés au Canada, 65,7\% étaient originaires d'un pays où le $\mathrm{VIH}$ est endémique.

En 2019, les notifications de santé publique d'Immigration, Réfugiés et Citoyenneté Canada étaient le plus souvent envoyées en Ontario (35,7\%), au Québec (28,0 \%), en Alberta $(18,9 \%)$ et en Colombie-Britannique $(8,4 \%)$.

\section{Programme de surveillance périnatale du VIH au Canada}

Selon le Programme de surveillance périnatale du VIH au Canada, 250 nourrissons ont été exposés au VIH durant la période périnatale au Canada en 2019. Un nourrisson a été déclaré positif au VIH en 2019. Ce nourrisson était asymptomatique et né d'une mère qui n'avait pas reçu de traitement antirétroviral prophylactique antepartum ou intrapartum. Depuis 2012, il y a eu en moyenne 250 expositions périnatales par an (étendue: 217 à 268) avec une moyenne de 5,5 infections confirmées par an (étendue: 1 à 12). La tendance de la proportion de mères séropositives au $\mathrm{VIH}$ recevant un traitement antirétroviral chaque année est en augmentation depuis 2015 (93,5\%), avec 96,2\% recevant un traitement antirétroviral en 2018 et $98,0 \%$ en 2019. 
En 2019, l'exposition la plus fréquemment signalée chez les mères séropositives au $\mathrm{VIH}$ a continué d'être le contact hétérosexuel $(77,0 \%)$, suivis par l'utilisation de drogues par injection (16,7\%). En ce qui concerne la race ou l'origine ethnique des mères, la plus souvent citée était noire $(58,4 \%)$. Viennent ensuite les mères s'identifiant comme autochtones $(20,4 \%)$ et blanches $(13,2 \%)$. La plupart des mères séropositives au $\mathrm{VIH}$ étaient d'origine africaine $(48,0 \%)$ ou nord-américaine $(34,8 \%)$.

\section{Discussion}

Au total, 2122 diagnostics de VIH ont été signalés en 2019 au Canada, et le taux de diagnostic national était de 5,6 pour 100000 habitants. Au cours de la dernière décennie, les taux sont restés stables, avec quelques fluctuations mineures. Le taux de diagnostic de 2019 est légèrement inférieur à celui de 2018; il faudra plus de temps et de données pour déterminer si cette baisse est le début d'une tendance continue.

Au total, 1188 migrants ont reçu un diagnostic positif pour le VIH lors d'un examen médical aux fins de l'immigration en 2019. Parmi ces cas, $52,7 \%$ ont été testés au Canada et $47,3 \%$ ont été testés à l'étranger avant leur arrivée au Canada. En 2019, 250 nourrissons ont été exposés au VIH durant la période périnatale au Canada. La seule transmission durant la période périnatale du VIH documentée concernait une mère qui n'avait pas reçu de traitement antirétroviral prophylactique antepartum ou intrapartum.

Comme les années précédentes, la plus grande proportion de nouveaux diagnostics de $\mathrm{VIH}$ est celle des hommes gbHARSAH et représente plus de la moitié des cas masculins adultes (56,2\%) en 2019. Une légère diminution des taux a été constatée chez les hommes en général depuis 2016 et des diagnostics de $\mathrm{VIH}$ chez les hommes gbHARSAH depuis 2017. Cette diminution des taux chez les hommes dans l'ensemble coïncide avec l'approbation de la prophylaxie pré-exposition par Santé Canada, et pourrait tenir compte de l'impact de cette nouvelle technologie de prévention du VIH dans cette population. Cette tendance correspond à celle observée dans d'autres pays développés, dont l'Australie et le Royaume-Uni $(14,15)$. En Australie, on observe une diminution de $25,4 \%$ du nombre de diagnostics de VIH chez les hommes gbHARSAH depuis 2016. Au Royaume-Uni, on observe une diminution de $47,1 \%$ depuis 2014 , un changement attribué à une baisse significative des diagnostics de VIH chez les hommes homosexuels et bisexuels. Au fur et à mesure de l'augmentation de l'utilisation de la prophylaxie pré-exposition dans les populations admissibles, on peut s'attendre à de nouvelles réductions du diagnostic du $\mathrm{VIH}$.

La diminution du nombre de diagnostics de VIH au Canada n'a pas été aussi importante que celle observée dans d'autres pays. Cela indique que l'on peut faire davantage pour accroître la sensibilisation et l'utilisation de la prophylaxie pré-exposition.
Sur la base des résultats d'une enquête menée en 2017 auprès des hommes gbHARSAH au Canada, 51,7 \% des participants ont déclaré qu'ils étaient susceptibles d'utiliser la prophylaxie pré-exposition si elle était abordable et disponible, et seulement $8,4 \%$ utilisaient la prophylaxie pré-exposition au moment de l'enquête (16).

Les taux de diagnostic chez les femmes ont légèrement augmenté depuis 2015. Cette tendance coïncide avec l'augmentation des cas de syphilis infectieuse chez les femmes (17). Ces tendances générales suggèrent une augmentation de la consommation de substances, de l'utilisation de drogues injectables et de la prévalence des ITTS dans certains réseaux de femmes à risque pour les ITSS. Ces tendances apportent également un soutien supplémentaire à l'approche nationale intégrée définie par le cadre du gouvernement du Canada (4) pour réduire l'impact sanitaire des ITSS au Canada dans les populations clés touchées par des épidémies qui se chevauchent (i.e. des syndémies) (18). Cette augmentation des taux chez les femmes n'a pas été observée dans les autres pays développés pour lesquels des renseignements étaient disponibles. En fait, les taux chez les femmes ont diminué aux États-Unis entre 2014 et 2018, à l'exception d'une légère augmentation ( $8 \%$ ) depuis 2014 du nombre de cas chez les femmes utilisatrices de drogues par injection (19). De même, en Australie, les taux de diagnostics de $\mathrm{VIH}$ chez les femmes ont légèrement diminué depuis 2017 (14), tandis qu'au RoyaumeUni, le nombre de nouveaux diagnostics de VIH chez les femmes a diminué de façon constante depuis 2010 (15).

Près d'un quart des diagnostics de VIH en 2019 ont été attribués aux peuples autochtones, ce qui indique une surreprésentation de cette population dans les données canadiennes sur le VIH. Étant donné que seul un nombre limité de provinces et de territoires font état de l'indigénéité, ces proportions sont probablement biaisées. Cependant, il est clair que les peuples autochtones sont surreprésentés parmi les personnes vivant avec le $\mathrm{VIH}$. De nouvelles estimations, qui s'appuient sur les données du Système national de surveillance du VIH/sida, ainsi que sur d'autres sources de données, indiquent que les infections parmi les populations autochtones représentaient $14 \%$ de toutes les nouvelles infections en 2018 , alors que les populations autochtones ne représentaient que $4,9 \%$ de la population canadienne totale (2).

Les données d'Immigration, Réfugiés et Citoyenneté Canada indiquent que si la proportion de migrants ayant obtenu un résultat positif au test VIH lors de leur examen médical aux fins de l'immigration est restée relativement stable ces dernières années, le nombre global de personnes qui émigrent au Canada a augmenté. Cependant, le nombre de diagnostics de VIH identifiés par les examens médicaux aux fins de l'immigration ne représente pas nécessairement les nouveaux cas de $\mathrm{VIH}$ au Canada. Certains migrants qui ont reçu des tests positifs au VIH lors d'examens médicaux aux fins de l'immigration à l'étranger peuvent ne pas arriver au Canada, et ceux établis lors d'examens 
médicaux aux fins de l'immigration au Canada peuvent déjà être pris en compte dans les rapports des provinces et territoires.

En outre, il serait difficile de déterminer le moment où le VIH a été contracté par les 626 migrants qui ont reçu un diagnostic positif lors des examens médicaux aux fins de l'immigration au Canada en 2019. II faut davantage de renseignements pour mieux comprendre l'épidémiologie du VIH chez les nouveaux Canadiens, en particulier ceux qui viennent de pays où le VIH est endémique.

En 2019, 250 nourrissons ont été exposés au VIH durant la période périnatale au Canada. Une seule transmission du $\mathrm{VIH}$ documentée concernait une mère qui n'avait pas reçu de traitement antirétroviral prophylactique antepartum ou intrapartum. Au fil des ans, d'importantes mesures d'atténuation ont été prises au Canada pour prévenir la transmission du VIH de la mère à l'enfant. Il s'agit notamment d'un meilleur accès aux soins prénataux, du dépistage systématique du VIH chez les femmes enceintes et de la disponibilité de traitements pour les mères séropositives au $\mathrm{VIH}$. Néanmoins, des occasions de prévention manquées continuent de se produire, principalement dans les populations vulnérables, ce qui entraîne un petit nombre d'infections durant la période périnatale (20).

Malgré les progrès réalisés dans la prévention, le diagnostic et le traitement du $\mathrm{VIH}$, le $\mathrm{VIH}$ et les autres ITTS représentent toujours un problème de santé important au Canada. Les données de surveillance, telles que celles présentées dans ce rapport, sont un élément clé pour comprendre le fardeau des ITSS au Canada et pour suivre les progrès du Canada vers les objectifs fixés dans le cadre (4).

\section{Forces et faiblesses}

La force principale de ce rapport repose dans le fait qu'il s'agit de la seule source de données épidémiologiques nationales sur tous les diagnostics déclarés de $\mathrm{VIH}$ au Canada. II intègre également des données sur le diagnostic du VIH chez les migrants au Canada et sur la transmission du VIH durant la période périnatale, ce qui permet de dresser un tableau plus complet de l'état du VIH au Canada.

Les faiblesses du Système national de surveillance du $\mathrm{VIH} / \mathrm{sida}$ ont été décrites précédemment $(10,12)$ et sont communes à la plupart des systèmes de surveillance. Bien qu'il soit difficile de déterminer les facteurs qui peuvent contribuer aux fluctuations constatées, les changements dans les pratiques de déclaration des autorités sanitaires des provinces et des territoires peuvent avoir eu un impact.

Le faible taux de réponse lié aux éléments de données liés à la race ou l'origine ethnique et au milieu d'expositions des nouveaux cas de $\mathrm{VIH}$, et les biais potentiels qui en résultent dans les données disponibles, créent des difficultés pour faire des inférences sur les facteurs qui influencent la transmission du VIH au Canada. L'Agence continue de travailler avec ses partenaires de surveillance pour améliorer la collecte des éléments de données, y compris les informations sur la race ou l'origine ethnique.

Comme le rapportent Popovic et al. (21), les cas de VIH déclarés par les provinces et territoires par l'entremise des mécanismes de surveillance de routine peuvent avoir été diagnostiqués antérieurement, soit dans une autre province ou un autre territoire, soit dans un autre pays; ces cas ont des incidences sur les tendances observées dans les taux de diagnostic du $\mathrm{VIH}$. Il est donc important de comprendre le fardeau global des diagnostics de VIH chez les personnes vivant actuellement au Canada.

\section{Conclusion}

Les données contenues dans ce rapport sont considérées comme provisoires et peuvent être modifiées dans les futurs rapports de surveillance du $\mathrm{VIH}$. S'il existe des divergences entre les données résumées dans ce rapport et les rapports des provinces et des territoires, il convient d'utiliser le rapport provincial et territorial le plus récent.

\section{Déclaration des auteurs}

N. H. - Conceptualisation, recherche, rédaction, ébauche initiale, ébauche finale, révision, correction, validation de données, visualisation, supervision

A. W. - Validation des données, ébauche initiale, correction, recherche

A. R. - Gestion des données, validation des données, recherche S. T. - Révision, correction

\section{Intérêts concurrents}

Aucun.

\section{Remerciements}

L'Agence de la santé publique du Canada (l'Agence) tient à remercier les personnes suivantes des programmes provinciaux et territoriaux de lutte contre le $\mathrm{VIH} /$ sida pour leur contribution et leur participation : S. Fathima, ministère de la Santé de I'Alberta; A. O’Brien, ministère de la Santé de l'Alberta; J. Wong, Service de prévention clinique, Surveillance et épidémiologie, Centre for Disease Control de la Colombie-Britannique; C. Loeppky, ministère de la Santé du Manitoba; R. RaaFat Gad, ministère de la Santé du Nouveau-Brunswick; S. Landsburg, ministère de la Santé du Nouveau-Brunswick; S. Wertz, ministère de la Santé du Nouveau-Brunswick; B. Halfyard, Santé et services communautaires, Direction générale de la santé de la population, Terre-Neuve-et-Labrador; M. O’Driscoll, Santé et services communautaires, Direction générale de la santé de la population, Terre-Neuve-et-Labrador; H. Hannah, ministère de la Santé 
et des Services sociaux, Territoires du Nord-Ouest; B. Billard, ministère de la Santé et du Mieux-être de la Nouvelle-Écosse; S. Fleming, ministère de la Santé et du Mieux-être de la Nouvelle-Écosse; K. Kullerperuma, ministère de la Santé du Nunavut; A. Kroch, Réseau ontarien de traitement du VIH; J. Liu, Santé publique Ontario; M. Murti, Santé publique Ontario; M. Cameron, Santé et Mieux-être, Île-du-Prince-Édouard; C. Cheverie, Santé et Mieux-être, Île-du-Prince-Édouard; $\mathrm{R}$ Bitera, Direction des risques biologiques et de la santé au travail, Institut national de santé publique du Québec; P.H. Minot, Direction des risques biologiques et de la santé au travail, Institut national de santé publique du Québec; B. Serhir, Laboratoire de santé publique du Québec, Institut national de santé publique du Québec; D. Sylvain, Laboratoire de santé publique du Québec, Institut national de santé publique du Québec; $H$. Bangura, ministère de la Santé de la Saskatchewan; J. Manalo, ministère de la Santé de la Saskatchewan; L. Strudwick, Contrôle des maladies transmissibles Yukon; S. Plitt, l'Agence, Alberta; E. Wong, I'Agence, Colombie-Britannique; J. Paul, l'Agence, Manitoba; A. Sullivan, I'Agence, Ontario; T. Schellenberg, I'Agence, Saskatchewan; M. Nichols, l'Agence, Nouvelle-Écosse; A. Bitnun et L. Sauvé, Programme de surveillance périnatale du VIH au Canada; C. Rank, A. Grundy et C. Nwoke, Immigration, Réfugiés et Citoyenneté Canada.

\section{Financement}

Cet article a reçu l'appui financier de l'Agence de la santé publique du Canada dans le cadre de son mandat de base.

\section{Références}

1. The Joint United Nations Programme on HIV/AIDS. UNAIDS data 2020. Geneva (CH): UNAIDS; 2020. https://www.unaids. org/sites/default/files/media_asset/2020_aids-data-book_en.pdf

2. Agence de la santé publique du Canada. Estimations de I'incidence et de la prévalence du $\mathrm{VIH}$, et des progrès réalisés par le Canada en ce qui concerne les cibles $90-90-90$ pour le VIH, 2018. Ottawa (ON) : ASPC; 2020 (accédé 2020-12-17). https://www.canada.ca/fr/sante-publique/services/publications/ maladies-et-affections/esume-estimations-incidence-prev alence-vih-progres-realises-canada-90-90-90.html

3. Agence de la santé publique du Canada. Rapport d'étape sur le $\mathrm{VlH} /$ sida et les populations distinctes : Personnes vivant avec le VIH/sida. Ottawa (ON) : ASPC; 2013 (accédé 2020-12-17). https://www.canada.ca/fr/sante-publique/services/vih-sida/ publications/rapports-etape-vRih-sida-populations-distinctes/ personnes-vivant-avec-vih-sida.html
4. Agence de la santé publique du Canada. Réduction des répercussions sur la santé des infections transmissibles sexuellement et par le sang au Canada d'ici 2030 : un cadre d'action pan-canadien sur les ITSS. Ottawa (ON) : ASPC; 2019 (accédé 2020-12-17). https://www.canada.ca/fr/ sante-publique/services/maladies-infectieuses/sante-sexuell e-infections-transmissibles-sexuellement/rapports-publications/ infections-transmissibles-sexuellement-sang-cadre-action.html

5. Agence de la santé publique du Canada. Accélérer notre intervention : plan d'action quinquennal du gouvernement du Canada sur les infections transmissibles sexuellement et par le sang. Ottawa (ON) : ASPC; 2019 (accédé 202012-17). https://www.canada.ca/fr/sante-publique/services/ rapports-publications/accelerer-notre-intervention -plan-action-quinquennal-infections-transmissibles -sexuellement-sang.html

6. Cohen MS, Chen YQ, McCauley M, Gamble T, Hosseinipour MC, Kumarasamy N, Hakim JG, Kumwenda J, Grinsztejn B, Pilotto JH, Godbole SV, Mehendale S, Chariyalertsak S, Santos BR, Mayer KH, Hoffman IF, Eshleman SH, Piwowar-Manning E, Wang L, Makhema J, Mills LA, de Bruyn G, Sanne I, Eron J, Gallant J, Havlir D, Swindells S, Ribaudo H, Elharrar V, Burns D, Taha TE, Nielsen-Saines K, Celentano D, Essex M, Fleming TR; HPTN 052 Study Team. Prevention of HIV-1 infection with early antiretroviral therapy. N Engl J Med 2011;365(6):493-505. DOI PubMed

7. Marks G, Crepaz N, Senterfitt JW, Janssen RS. Meta-analysis of high-risk sexual behavior in persons aware and unaware they are infected with HIV in the United States: implications for HIV prevention programs. J Acquir Immune Defic Syndr 2005;39(4):446-53. DOI PubMed

8. Metsch LR, Pereyra M, Messinger S, Del Rio C, Strathdee SA, Anderson-Mahoney P, Rudy E, Marks G, Gardner L; Antiretroviral Treatment and Access Study (ARTAS) Study Group. HIV transmission risk behaviors among HIV-infected persons who are successfully linked to care. Clin Infect Dis 2008;47(4):577-84. DOI PubMed

9. Antiretroviral Therapy Cohort Collaboration. Life expectancy of individuals on combination antiretroviral therapy in high-income countries: a collaborative analysis of 14 cohort studies. Lancet 2008;372(9635):293-9. DOI PubMed

10. Haddad N, Robert A, Weeks A, Popovic N, Siu W, Archibald C. Le VIH au Canada - Rapport de surveillance, 2018. Relevé des maladies transmissibles au Canada 2019;45(12):334-43. DOI

11. Agence de la santé publique du Canada. Définitions nosologiques des maladies transmissibles faisant l'objet $d$ 'une surveillance nationale. Résultats d'une consultation provinciale territoriale (PT). Relevé des maladies transmissibles au Canada 2009;35S2:S1-131. https://www.canada.ca/content/dam/ phac-aspc/migration/phac-aspc/publicat/ccdr-rmtc/09pdf/35s2fra.pdf

12. Agence de la Santé Publique du Canada. Le VIH et le sida au Canada - Rapport de surveillance en date du 31 décembre 2014. Ottawa (ON) : ASPC; 2015. https://www.canada.ca/fr/ sante-publique/services/publications/maladies-et-affections/ vih-et-sida-canada-rapport-surveillance-31-decembre-2014.html

13. Statistique Canada. Estimations démographiques annuelles: Canada, provinces et territoires, 2019. Ottawa (ON) : Statistique Can; 2019 (accédé 2020-12-17). https://www150.statcan.gc.ca/ n1/pub/91-215-x/91-215-x2019001-fra.htm 
14. The Kirby Institute. National HIV notifications. Q1 2015-Q4 2019. Kensington (AU): The Institute; 2019. https://kirby. unsw.edu.au/sites/default/files/kirby/report/National-HI V-Quarterly-Report_2019-Q4.pdf

15. Public Health England. Trends in HIV testing, new diagnoses and people receiving HIV-related care in the United Kingdom: data to the end of December 2019. Health Protection Report 2020;14(20):1-15. https://assets.publishing.service.gov.uk/ government/uploads/system/uploads/attachment_data/ file/939478/hpr2020_hiv19.pdf

16. Brogan N, Paquette DM, Lachowsky NJ, Blais M, Brennan DJ, Hart TA, Adam B. Résultats canadiens de l'Enquête européenne en ligne sur la sexualité entre hommes (EMIS-2017). Relevé des maladies transmissibles au Canada 2019;45(11):298-310. DOI

17. Syphilis infectieuse au Canada, 2018. Infographique Relevé des maladies transmissibles au Canada 2020;45(11):332.

https://www.canada.ca/content/dam/phac-aspc/documents/ services/reports-publications/canada-communicabl e-disease-report-ccdr/monthly-issue/2019-45/issue-11november-7-2019/ccdrv45i11a05f-fra.pdf

18. Murti M, Wong J, Whelan M, Renda C, Hohenadel K, Macdonald L, Parry D. Nécessité d'une surveillance intégrée en matière de santé publique pour aborder les syndémies d'infections transmissibles sexuellement et par le sang. Relevé des maladies transmissibles au Canada 2019;45(2-3):68-72. DOI
19. Centers for Disease Control and Prevention. Diagnoses of HIV infection in the United States and Dependent Areas. HIV Surveillance Report. Atlanta (GA): National Center for HIV/AIDS, Viral Hepatitis, STD, and TB Prevention, CDC; 2018 (modifié 2020-05-07; accédé 2020-09-01). https://www.cdc.gov/hiv/ library/reports/hiv-surveillance/vol-31/index.html

20. Bitnun A, Lee T, Brophy J, Samson LM, Kakkar F, Vaudry W, Tan B, Money DM, Singer J, Sauvé LJ, Alimenti A; Canadian Perinatal HIV Surveillance Program. Missed opportunities for prevention of vertical HIV transmission in Canada, 1997-2016: a surveillance study. CMAJ Open 2018;6(2):E202-10. DOI PubMed

21. Popovic N, Yang $Q$, Haddad N, Weeks A, Archibald C. Améliorer la surveillance nationale des nouveaux diagnostics de $\mathrm{VIH}$. Relevé des maladies transmissibles au Canada 2019;45(12):344-7. DOI 


\section{Annexe : Liste des tableaux supplémentaires}

Ces tableaux sont disponibles sur demande à : phac.hass.aspc@ canada.ca

Tableau S1 : Taux de diagnostics du VIH (pour 100000 habitants) par province ou territoire et année de diagnostic (tous les âges)

Tableau S2 : Taux de cas de VIH (tous les âges) par province ou territoire, sexe et année du diagnostic - Canada, 1985 à 2019

Tableau S3 : Nombre de cas de VIH par groupe d'âge et province ou territoire - Canada, 2017 à 2019

Tableau S4 : Nombre cumulatif de cas de VIH chez les adultes (15 ans ou plus) et les enfants (moins de 15 ans) par sexe Canada, 1985 à 2019

Tableau S5 : Nombre de cas de VIH chez les adultes (15 ans ou plus) par année de diagnostic et par sexe - Canada, 1985 à 2019

Tableau S6 : Nombre de cas de VIH par groupe d'âge, sexe et année du diagnostic - Canada, 1985 à 2019

Tableau S7 : Nombre et répartition en pourcentage de cas de VIH chez les adultes (15 ans ou plus) par catégorie d'exposition et année du diagnostic - Canada, 1985 à 2019

Tableau S8 : Nombre et répartition en pourcentage de cas de $\mathrm{VIH}$ chez les hommes adultes (15 ans ou plus) par catégorie d'exposition et année du diagnostic - Canada, 1985 à 2019

Tableau S9 : Nombre et répartition en pourcentage de cas de $\mathrm{VIH}$ chez les femmes adultes (15 ans ou plus) par catégorie d'exposition et année du diagnostic - Canada, 1985 à 2019

Tableau S10 : Nombre et répartition en pourcentage de cas de $\mathrm{VIH}$ chez les adultes (15 ans ou plus) par catégorie d'exposition et groupe d'âge-Canada, 2018 à 2019
Tableau S11 : Nombre et répartition en pourcentage de cas de VIH chez les enfants (15 ans ou moins) par catégorie d'exposition et année du diagnostic - Canada, 1985 à 2018

Tableau S12 : Nombre de cas de VIH par catégorie d'exposition et par province ou territoire - Canada, 2018 à 2019

Tableau S13 : Nombre et répartition en pourcentage des candidats à l'immigration au Canada ayant reçu un diagnostic de VIH à la suite d'un examen médical aux fins de l'immigration par année - 2002 à 2019

Tableau S14 : Nombre et répartition en pourcentage des candidats à l'immigration au Canada ayant reçu un diagnostic de VIH à la suite d'un examen médical aux fins d'immigration, par sexe, groupe d'âge et province-2012 à 2019

Tableau S15 : Nombre de nourrissons exposés au VIH en période périnatale par année de naissance, statut actuel et utilisation de la thérapie antirétrovirale à des fins prophylactiques - Canada, 1984 à 2019

Tableau S16 : Nombre de nourrissons périnataux exposés au VIH par catégorie d'exposition maternelle et année de naissance du nourrisson - Canada, 1984 à 2019

Tableau S17 : Nombre de nourrissons périnataux exposés au VIH, par statut ethnique et statut d'infection - Canada, 1984 à 2019

Tableau S18 : Nombre de nourrissons périnataux exposés au VIH par pays de naissance de la mère et par statut d'infection Canada, 1984 à 2019

Tableau S19 : Nombre de nourrissons exposés au VIH par voie périnatale, par région géographique et par statut lors du dernier rapport - Canada, 1984 à 2019

Tableau S20 : Statistiques internationales sur les cas de VIH signalés - Canada, 2018 